\title{
Externally prestressed monolithic and segmental concrete beams under torsion: A comparative finite element study.
}

\begin{abstract}
Externally Prestressed segmental beams are widely used in the construction of bridge structures today. These beams have many attractive advantages for rehabilitation and strengthening of existing structures as well. However, segmental beams experience significant effect under combined stresses at the joint interfaces between segments. This paper presents a finite element study on the effect of torsion on the structural behavior of both external prestressed monolithic and segmental concrete beams. Geometric and material nonlinearities were included in the study. The results show significant difference in the behavior of monolithic and segmental beams under torsion.
\end{abstract}

Keyword: Bridge structures; Combined stress; Concrete beam; Existing structure; Finiteelement study; Geometric and material nonlinearities; Joint Interfaces; Prestressed; Structural behaviors; Bridges 